\title{
Synthesis and Evaluation of the Antidiabetic Potentials of Chitosan Combinations with Metformin and Momordica Balsamina Leaf Extract in Alloxan-Induced Diabetic Rats
}

\author{
Nafisatu Kabir ${ }^{* *}$, Aliyu Sunusi Aliyu², Jibrin Iliyasu Jibrin² \\ ${ }^{1}$ Department of Biochemistry, \\ Federal University Dutse \\ Jigawa State \\ ${ }^{2}$ Department of Microbiololgy and Biotechnology, \\ Federal University Dutse \\ Jigawa State
}

Email: kabirnafisa0@yahoo.com

\begin{abstract}
Chitosan, a carbohydrate biopolymer, is medically gaining attention as a dietary supplement and a drug beneficial for improving blood glycemia, impaired glucose tolerance and altered lipid metabolism. Largely obtained from crustaceans, chitosan is also a promising vehicle used to deliver anti-diabetic drugs in implantable as well as injectable and oral routes. The present study is therefore aimed to synthesize chitosan and evaluate its antidiabetic potentials in combination with metformin and/or methanolic leaf extract of Momordica balsamina (MBM) in alloxan-induced diabetic rats. Chitosan was synthesized from crayfish shell through demineralization, deproteinization, deacetylation, and decolouration of the produced chitin. In the present study, a simple and an inexpensive acid-base titrimetric method was used for the determination of degree of deacetylation while the physicochemical properties; moisture content and ash content were determined using standard procedures. The efficacy of the drug binary systems of chitosan-metformin and chitosan-MBM were evaluated on the glucose tolerance of Wistar Albino rats made diabetic by a single interperitoneal injection of alloxan monohydrate $(150 \mathrm{mg} / \mathrm{kg}$ body weight). The oral administration of both drug binary systems and chitosan exerted a significant $(p<0.05)$ improvement on carbohydrate metabolism via glucose tolerance test when compared to the untreated diabetic rats. The blood glucose level of the chitosan group returned to near normal level 2 hours post-porandially, while chitosan-MBM, chitosanmetformin and metformin groups returned to their initial values only after 4 hours. Our findings are suggestive of the fact that chitosan-based drug binary systems possess anti-hyperglycemic activities and thus the potential to improve the pharmacokinetic and pharmacological profile of oral hypoglycemic drugs/herbs. Our findings could serve as basis for the development of novel antidiabetic drug systems.
\end{abstract}

Keywords: Antidiabetic, Chitosan, Metformin, Methanolic extract, Momordica balsamina 


\section{INTRODUCTION}

Diabetes mellitus (DM) is a metabolic disorder characterized by defect in insulin secretion, insulin action or both. Deficiency of insulin or failure of response of its receptors plays a central role in the pathophysiology of both type 1 and type 2 diabetes mellitus (Shoback et al., 2011). DM is associated with impaired beta cell function, elevated insulin resistance and blood glucose concentrations. These factors lead to an increased risk of macro- and microvascular circulations that are in turn, associated with the five classic complications of DM, i.e. retinopathy, neuropathy, nephropathy, cardiovascular complications, and delayed wound-healing (Mealey et al., 2006) especially in uncontrolled glycemia. Glucose tolerance test is a method used to diagnose instance of especially type 2 and gestational diabetes mellitus, insulin resistance and impaired beta cell function (Shoback et al., 2011).

The current available oral treatments for type 2 diabetes mellitus include sulfonylureas, biguanides, thiazolinediones and alpha glucosidase inhibitors. Metformin is the most common biguanide used as a first line drug for type 2 diabetes mellitus management. However, metformin has a low degree of bioavailability (50-60\%) and short half-life (0.9- 2.6 hours) thus requiring frequent administration at high doses (Hajjar et al., 2013). Unfortunately, at higher doses or with long term usage, it is often associated with several adverse effects such as lactic acidosis, diarrhea, nausea, vomiting and flatulence (Scheen and Paquot, 2013). Furthermore, owing to its short half-life, rapid clearance and inability to reach its desired site, it fails to achieve the desired pharmacological effect. This could be due to its hydroxylation in the stomach with gastric acids where they get digested by specific enzymes before exerting its pharmacological action (Yang et al., 2018). Therefore, drug transport vehicles to circumvent these drug delivery problems are required.

Plants have always been very good sources of drugs and many of the currently available drugs have been derived directly or indirectly from them. Several herbs have shown antidiabetic activity when evaluated using different in vitro and in vivo experimental techniques. In North-West Nigeria, traditional herbal healers use Momordica balsamina as one of the major components of antidiabetic formulations because of its availability and documented multiple bioactivity. The leaf and fruit extracts of Momordica balsamina is documented to have antiplasmodial and antiviral activity as well as shigellocidal, antidiarrhoeal, antiseptic, antibacterial, antiviral, antinflammatory, hypoglycemic and antimicrobial properties (Akinyemi et al., 2005; Bot et al., 2007). The leaves are important source of nutrients having 17 amino acids (Hassan and Umar, 2006) as such could be promoted as a protein supplement for cereal-based diets in poor rural communities. The phytochemicals secreted from the fruit and leaves of Momordica balsamina are resins, alkaloid, flavonoid, anthroquinone, glycosides, steroids, terpenes, cardiac glycoside, saponins and carbohydrate (Bot et al., 2007). Due to inherent side effects, cost, unavailability and contraindications associated with modern oral hypoglycemic drugs many patients have opted for traditional herbal medicines in combination with the modern drugs to control hyperglycemia and prevent long term complications. Hence the need to improve the solubility, oral bioavailability and delivery modes of herbal formulations as a pathway of producing novel conventional drugs.

Chitin is the second most abundant polysaccharide in nature after cellulose and largely exist in the exoskeletons of shelled mollusks, crustaceans, insects, yeast and few species of fungal cell walls (Santa et al., 2017). Chitin is a bio polymeric substance derived from a homopolymer of $\beta(1 \rightarrow 4)$ linked N-acetyl-D-glucosamine. Commonly, the shell of selected crustaceans consists of $30-40 \%$ protein, $30-50 \%$ calcium carbonate and calcium phosphate 
and $20-30 \%$ chitin (Knorr, 1984). Chitin can be used to produce chitin-derived products, such as chitosans, chito-oligosaccharides and glucosamine, but also for bioplastic production. Most of the naturally occurring polysaccharides, for instance, cellulose, pectin, alginic acid, and carrageenan are neutral or acidic in nature, whereas chitin and chitosan are examples of highly basic polysaccharides (Kato et al., 2003). Chitosan is a polysaccharide made up of deacetylated $\beta$-(1-4) D-glucosamine and acetylated units of N-acetyl-Dglucosamine. Its exceptional biological properties have made it an excellent candidate for applications in cosmetics, medicine, pharmacy, agriculture, in the preservation of agricultural commodities in the food industry and waste water treatment (No et al., 1999) among many other industrial applications. The biomedical application of chitosan includes its use in rapid clotting of blood, in bandages and weight loss (Kumar et al., 2017). Chitosan is also claimed to be used for wound healing, cancer, as anti-thrombogenic and heamostatic materials, vaccine adjuvant and for antimicrobial, antioxidant and antitumor activity (EliehAli-Komiand Hamblin, 2016). Other industrial and medical application of chitosan covers many areas such as chromatographic separation, tissue engineering, ophthalmological application and drug delivery system (Dutta et al., 2004).

Formation of micro and nanoparticles using chitosan as a biomaterial is also being considered by many researchers for the formulation of pharmaceutical drugs and to develop new binary polymeric systems in order to achieve improved efficacy and pharmacological profile of oral drugs. The unique properties of chitin and chitosan that allow for its medical and pharmacological relevance include polyoxysalt formation, ability to form films, chelation with metal ions and optical structural characteristics (Kumar, 2000). Owing to its biocompatibility, biodegradability, structural variability and non-toxicity, conjugating Momordica balsamina and/or metformin with chitosan could be a promising excipient for oral antidiabetic therapeutics. This could therefore facilitate transmucosal absorption, bioavailability, increase stability, reduce side effects and retain the drug locally to permit an extended time for drug absorption to effectively treat diabetes and its complications. Furthermore, despite the lack of clinical evidence for efficacy, chitosan dietary supplements are currently receiving scientific attention as a result of health claims that they can block fat absorption for weight loss, improve glycemia and glucose resistance when used by diabetic patients. However, these claims are unsubstantiated and would require to be validated in pre- clinical settings. The present study therefore aims to produce chitosan and characterize it using a cheap titrametric method which is more affordable and available in sub-Saharan Africa than conventional FTIR. In addition, evaluation of the antidiabetic potential of the chitosan produced in combination with Momordica balsamina (antidiabetic plant) and metformin in alloxan induced diabetic rats could scientifically validate it's claimed health benefits.

\section{METHODOLOGY}

\section{Raw Materials Collection and Preparation}

Shrimp shells were obtained from a local market in Kano (Yankura, Sabon Gari Market). Upon purchase, shells of tail and the head were separated, ground, sieved and kept in a labeled container until required. Conversely, fresh Momordica balsamina leaves were collected from Dutse, Jigawa state. The leaves were identified and authenticated by a botanist in the Department of Biological Sciences, Federal University Dutse. Dust particles were washed from the leaves with borehole water and shade-dried for two weeks. The dried leaves were grounded to a fine powder using mortar and pestle and stored in air tight containers prior to extract preparation. The grounded leaves $(2 \mathrm{~g})$ were extracted with $1000 \mathrm{mLs}$ of methanol by 
continuous percolation using rotary evaporator. A green colored methanolic extract of Momordica balsamina (MBM) was obtained.

\section{Production of Chitosan}

Chitosan was synthesized using the method described by Gunawan (2018) and FernandezKim, (2004). Dilute hydrochloric acid (1M), $\mathrm{NaOH}(1 \mathrm{M}), 12 \mathrm{M} \mathrm{NaOH}$ and absolute ethanol were used for demineralization, deprotenization, deacetylation and decolorization of the prepared shrimp shells respectively. The product obtained is chitosan.

\section{Determination of Percentage Yield of Chitosan}

The percentage yield was determined by comparing weight measurements of the raw material and that of the chitosan obtained after treatment.

Yield of chitosan $(\%)=($ Extracted chitosan $(\mathrm{g}) /$ Crayfish shells $(\mathrm{g})) \times 100$

\section{Moisture and Ash contents determination}

Ash and moisture contents (\%) were evaluated using standard ashing method described by AOAC (2005) and Hossain et al. (2019).

\section{Determination of Degree of Deacetylation (DD)}

Degree of Deacetylation was determined using acid-base titration method (Domard and Rinaudo, 1983). Chitosan $(0.1 \mathrm{~g})$ was dissolved in $30 \mathrm{ml} \mathrm{HCl}$ aqueous solution $(0.1 \mathrm{M})$ at room temperature. Exactly five (5) drops of methyl orange indicator was added. The red chitosan solution was titrated with $0.1 \mathrm{M} \mathrm{NaOH}$ solution until it turned orange.

The DD \% was calculated by the formula:

$\mathrm{C}_{1}=$ concentration of standard HCL $(\mathrm{mol} / \mathrm{L})$

$$
D D \%=\frac{C_{1} V_{1}-C_{2} V_{2}}{M \times 0.0994} \times 0.016
$$

$\mathrm{C}_{2}=$ standard $\mathrm{NaOH}$ solution $(\mathrm{mol} / \mathrm{L})$

$\mathrm{V}_{1}=$ volume of standard HCL aqueous solution used to dissolved chitosan (ml)

$\mathrm{V}_{2}=$ volume of standard $\mathrm{NaOH}$ solution consumed during titration $(\mathrm{ml})$

$\mathrm{M}=$ weight of chitosan (g)

0.016 is a Standard value

\section{Phytochemical Screening of MBM}

MBM was screened qualitatively for alkaloids, steroids, triterpenoids, flavonoids, tannins, carbohydrate and/or glycosides, saponins and phenols using different standard phytochemical tests as described by Sofowora (1993) and Trease and Evans (2002).

\section{Experimental Animals and Induction of Diabetes}

Healthy adult albino Wistar rats of both sexes weighing averagely 50-60 grams were used for the experiment. They were maintained under standard animal house conditions of 12 hours natural cycle of light and darkness following the NRC guidelines for use of experimental animals (1996).

Prior to the induction of diabetes, the rats were fasted overnight for 10 hours and freshly prepared alloxan in normal saline $(150 \mathrm{mg} / \mathrm{kg}$ body weight) was injected intraperitoneally. Four (4) hours after induction the rats were maintained on $5 \%$ D-glucose solution for $24 \mathrm{hrs}$. The rats were allowed to stabilize for 3 days and blood glucose was determined using blood obtained from rat tail vein. The rats with fasting blood glucose of $9.7 \mathrm{mmol} / \mathrm{L}$ were used for the study. 


\section{Experimental Design}

Diabetic rats were randomly assigned into 5 groups of 4 rats each. Treatment was administrated by gavage as follows:

Group 1- Normal rats, received distilled water: Normal control

Group 2- Diabetic rats, received distilled water: Diabetic control

Group 3- Diabetic rats, received $500 \mathrm{mg} / \mathrm{kg}$ body weight (bw) chitosan

Group 4- Diabetic rats, received Chitosan + Metformin binary system $(500 \mathrm{mg} / \mathrm{kg} \mathrm{bw})$

Group 4- Diabetic rats, received Chitosan-MBM binary system (500mg/kg body weight)

Group 5- Diabetic rats, received metformin (500 mg/kg bw)

\section{Glucose Tolerance Test}

Diabetic rats were given oral dose of glucose $(3 \mathrm{~g} / \mathrm{kg}), 30$ minutes post extract administration. Blood sample was collected from the tail vein at $0 \mathrm{hr}$ (prior extract administration), $1 \mathrm{hr}, 2 \mathrm{hr}$, $3 \mathrm{hr}$ and $4 \mathrm{hr}$ for blood glucose determination using glucometer (Accuchek, Germany).

\section{Statistical Analysis}

Values obtained were recorded as mean \pm standard deviation using Microsoft excel package. ANOVA and Tukey's posthoc test was used to determine difference between groups. Graph of A plot glucose tolerance curve was made using the glucose concentrations obtained against time of sampling and area under the curve was determined.

\section{RESULTS}

The chitosan produced with $75.44 \%$ degree of deacetylation was light brown in color and the percentage yield was $12.10 \%$. The moisture and ash content of the chitosan was $9.12 \%$ and $0.95 \%$ respectively (Table 1 ).

Table1: Characterization of Chitosan Produced from Crayfish Shell

\begin{tabular}{cc}
\hline Parameters & Percentage (\%) \\
\hline Colour (powdered) & Light Brown \\
Percentage Yield & 12.10 \\
Moisture Content & 9.12 \\
Ash Content & $0.95 \%$ \\
Degree of Deacetylation & $75.44 \%$ \\
\hline
\end{tabular}

As shown in Table 2, the phytochemical screening of the crude methanolic extract of momordica balsamina revealed the presence of alkaloid, antraquinones, saponins, phenols, terpenoids, flavonoids, tannins, steroids, and absence of phlobatanins, resins and cardiac glycosides.

Table 2: Some Phytochemical Constituents of Methanolic Extract of Momordica balsamina

\begin{tabular}{clc}
\hline CHEMICAL & TEST & MBM \\
CONSTITUENTS & & + \\
\hline ALKALOIDS & Mayer's & + \\
& Dragendroff's & + \\
& Wagner's & -
\end{tabular}




\begin{tabular}{llc}
\hline ANTHRAQUINONES & Borntrager's & + \\
SAPONINS & Frothing & + \\
PHENOLS & Pyridins-Ferric Chloride & + \\
TERPENOIDS & Libermann-Burchard & + \\
FLAVONOIDS & Sodium hydroxide & + \\
RESINS & Acetic anhydride & + \\
TANNINS & Ferric Chloride & \\
& & + \\
STEROIDS & & \\
& Libermann-Burchard & - \\
PHLOBATANNINS & Sulphuric acid & \\
\hline
\end{tabular}

$(+)=$ Present $(-)=$ Absent; MBM- Methanolic extract of Momordica balsamina

As shown in Table 3, the mean blood glucose level of the chitosan treated group increased from an initial blood glucose level of $9.73 \pm 0.42$ at 0 hour to $14.72 \pm 3.05$ at 1 hour which returned to the initial value of $9.31 \pm 1.52$ at 2 hours. Conversely a similar pattern of change in blood glucose was observed in the chitosan-MBM, chitosan-metformin and metformin groups with their blood glucose levels returning to the initial level in the $4^{\text {th }}$ hour (Table 3 ). When compared between groups, no statistical difference was observed in the blood glucose level between diabetic and chitosan-metformin group. However, significant difference $(\mathrm{p}<0.05)$ was observed between the chitosan-MBM, chitosan metformin and metformin group. Furthermore, comparison between chitosan treated group at 4 hours to the normal control group revealed no statistical difference (Table 3).

Table 3: Effect of MBM on Oral Glucose Tolerance of Alloxan-Induced Diabetic Rats

\begin{tabular}{llllll}
\hline \multicolumn{1}{c}{ Treatment } & 0 hour & $\begin{array}{c}1 \text { hour } \\
\text { Blood Glucose }\end{array}$ & $\begin{array}{c}2 \text { hours } \\
\text { mol/L) }\end{array}$ & 3 hours & 4 hours \\
\hline Normal Control & $6.47 \pm 0.80$ & $9.22 \pm 0.39$ & $7.23 \pm 1.11$ & $7.79 \pm 0.87$ & $6.85 \pm 1.13^{\mathrm{a}}$ \\
Diabetic Control & $9.72 \pm 0.39$ & $18.47 \pm 2.96$ & $17.72 \pm 1.47$ & $14.04 \pm 1.47$ & $11.13 \pm 0.97 \mathrm{c}$ \\
Chitosan & $9.73 \pm 0.42$ & $14.72 \pm 3.05$ & $9.31 \pm 1.52$ & $9.02 \pm 2.67$ & $7.43 \pm 1.36^{\mathrm{a}}$ \\
Chitosan-MBM & $10.13 \pm 1.27$ & $15.59 \pm 6.12$ & $12.75 \pm 1.40$ & $11.56 \pm 2.66$ & $9.62 \pm 2.00^{\mathrm{b}}$ \\
Chitosan-Metformin & $10.63 \pm 2.44$ & $17.16 \pm 4.72$ & $18.19 \pm 12.14$ & $16.15 \pm 4.78$ & $12.12 \pm 4.01^{\mathrm{c}}$ \\
Metformin & $10.22 \pm 1.07$ & $13.06 \pm 1.90$ & $15.97 \pm 5.29$ & $12.35 \pm 2.75$ & $8.41 \pm 1.32^{\mathrm{b}}$ \\
\hline
\end{tabular}

CMBM Chitosan-Momordicabalsaminacombination

Values expressed are mean \pm Standard deviation per group $(n=4)$.

Significant improvement $(\mathrm{p}<0.05)$ in glucose tolerance was observed in the chitosan, chitosan-metformin, chitosan-MBM and metformin treated diabetic rats as shown in Table 4. Furthermore, similar glucose tolerance response was observed $(p<0.05)$ between the chitosan and chitosan-MBM treated groups.

Table 4: Area under the glucose tolerance curve of chitosan and its drug binary systems in alloxan induced diabetic rats

\begin{tabular}{lc}
\hline Treatment & Area under the Glucose Tolerance Curve (mmol/L/hour) \\
\hline Normal Control & $45.53 \pm 5.29 \mathrm{a}$ \\
Diabetic Control & $180.00 \pm 48.30 \mathrm{e}$ \\
Chitosan & $104.45 \pm 43.27 \mathrm{~b}$ \\
CMBM & $106.43 \pm 20.04^{\mathrm{b}}$ \\
Chitosan-metformin & $108.29 \pm 9.01^{\mathrm{c}}$ \\
Metformin & $167 \pm 46.69 \mathrm{~d}$ \\
\hline CMBM Chitosan-Momordica balsamina Combination. Values expressed are mean \pm Standard Deviation in each \\
group ( $\mathrm{n}=4)$. Values within a column with different superscripts are statistically significant at $\mathrm{p}<0.005$
\end{tabular}




\section{DISCUSSION}

Drug administration is generally performed to treat disease progression and an ideal drug should be capable of reaching specific site of action and accumulating to its effective concentration. Many approaches have been developed and utilized for drug administration and the most common is oral administration which has been shown to be efficient, induces minimal side effect and easy to administer. Chitosan has been widely used in pharmaceutical industries for drug delivery systems in different forms including tablets, microspheres, micelles, vaccines, nucleic acids, hydrogels, nanoparticles and conjugates (Jabbal et al., 2012). Chitosan has been used for the release of insulin, gentamicin sulphate, diclofenac, clarithromycin, cimetidine, famotidine, bovine serum albumin, clozapine, ovalbumin, doxorubicin, ofloxacin, 5-fluorouracil (Bansal et al., 2011).

In the present study cray fish shell was used to produce a biotechnological product through demineralization, removal of protein and some trace amount of fats with the conversion of chitin in the shell to chitosan. This is achieved with a strong alkali such as sodium hydroxide. Chitin is converted to chitosan because sodium hydroxide has the ability to remove acetyl group at carbon number two of the chitin to become chitosan. Degree of deacetylation (DD) ranges from $56 \%$ to $99 \%$, depending on the source of chitin (species) and the method of preparation (Akakuru and Isiuku, 2017). This is in agreement with the quality (degree of deacetylation) of chitosan obtained in this study. Determination of the quality of chitosan produced is essential because, the main parameters influencing the characteristics of chitosan are its molecular weight and degree of deacetylation, which affect the solubility, rheological and physical properties (Khan, 2002). Degree of Deacetylation is further affected by other factors, such as the concentration of the alkali used in its production, the reaction temperature, the reaction time and the particle size (Akakuru and Isiuku, 2017). However, according to Li et al. (1992) for the desired solubility of chitosan, the deacetylation of chitin has to be achieved by at least $70 \%$ or above. The DD value is also crucial as it is important in assessing the application of chitosan produced in nutritional, medical and pharmacological industries (Kumirska et al., 2010). In addition, the method used for the determination of DD also determines the percentage value. In the present study a titrimetric method using methyl orangeas indicator was used. This method is demonstrated to be simple fast, widely adaptable and inexpensive which has the potential for widespread use especially in developing countries where FTIR and 1H NMR are expensive. Several techniques have been developed and assessed for DD of chitosan (Shigemasa et al., 1996; Kumirska et al., 2010). However, there is currently no standard method used by different research industries for DD evaluation thus results in variation of acceptable ranges.

The moisture and ash content of chitosan produced in this study complies with the findings of Sikder et al. (2019). High quality chitosan have been reported to have an ash content of less than 1\% (Sikder et al., 2019). Ash content is an indicator of the effectiveness of the demineralization step for removal of $\mathrm{CaCO}_{3}$. However, it is important to note that, elimination of the demineralization results in products having 31-36\% of ash content (Fernandez and Kim, 2004). Therefore, at $0.95 \%$ ash content the chitosan produced in the present studycan be said to be of high quality. Moisture content is an important factor for DD determination and moisture values of 5.8-6.0 \% were reported by Jiang et al. (2017).This finding is not consistent 
with that of the present study. Experimental errors could be a possible explanation of the difference observed.

In the present chitosan-metformin and chitosan-methanolic extract of Momordica balsamina produced a potent anti-hyperglycemic effect. The hydrophilic properties of chitosan and the methanolic extract could have facilitated the rapid entry of the herbal combination into the aqueous body environment more than the metformin. However, combination of metformin with chitosan might haveretained the drug locally to permit an extended time for drug absorption, thus pronounced decline in blood sugar began at 3 hours. In addition,the versatile properties of chitosan could have lend itself for microparticle formation of the chitosan binary system (Kumari and Rani, 2011). The release of drug from chitosan based dosage form depends upon the morphology, size, density, and extent of crosslinking of the particulate system, physico-chemical properties of drug as well as the polymer characteristics such as whether it is hydrophilic or hydrophobic, has gel formation ability, swelling capacity, mucoadhesive or bioadhesive properties and also on the presence of other excipients present in the dosage form (Bansal et al., 2011, Akakuru and Isiuku, 2017).As a drug carrier, chitosan and its derivatives improve drug absorption and stabilize drug constituents for drug targeting in addition to drug release enhancement (Jiang et al., 2004).

The major objective of use of antidiabetic herbs just like hypoglycemic drugs is to enhance the achievement of good glycemic control. The phytochemicals reported from the fruit and leaves of Momordicabalsaminaare similar to those observed in the present study. Combination of chitosan and the phytochemicals found in the leaves of Momordicabalsamina; reported to be responsible for its vast medicinal applications can be the reason for its efficacy. The antihyperglycemic effect of the extract-chitosan combination could be further augmented if purified phytochemicals were used.It is therefore recommended that Mormodicabalsaminashould be subjected to toxicological and phytochemical studies to identify toxic effects and bioactive principles. Further studies should be carried out to ascertain the mechanism of action of chitosan and the leaves of Mormodicabalsamina in exerting their medicinal functions. Chitosan-based micro and nano particles loaded with appropriate antidiabetic drugs should be formulated for good diabetes mellitus management.

\section{CONCLUSION}

The chitosan-metformin/Momordicabalsaminamethanolic leafextract drug binary systems demonstrated antidiabetic potentials as evidenced by an improved glucose tolerance. The influence of chitosan on both drug and extract were paramount as such the twobinary systems have thepotential to improve the pharmacokinetic and pharmacological profile of oral hypoglycemic drugs in addition to the biological activity of chitosan. Chitosan as a natural resource that is non- toxic, cheap and biocompatible, could form the basis for its use as an excipient during tablet formulation as well asdrug carrier especially in developing countries.

\section{ACKNOWLEDGEMENT}

We would like to thank MrYakubuOtaro of Department of Zoology, Bayero University Kano, for animal care and handling.

\section{Conflict of interest}

The authors declare no conflict of interest. 


\section{REFERENCES}

Akinyemi, K.O., Mendie, U.E., Smith, S.T., Oyefolu, A.O. and Coker, A.O. (2005). Screening of Some Medicinal Plants Used in South-West Nigerian Traditional Medicine for Anti-Salmonella Typhi Activity. Journal of Herbal Pharmacotherapy, 5(1):45-60.

AOAC. (2005). Official Method of Analysis. 18 th Edition, Association of Officiating Analytical Chemists, Washington DC, Method 935.14 and 992.24.

Bansal, V., Sharma, K.P., Sharma, N., Pal, P.O. and Malviya, R. (2011). Applications of Chitosan and Chitosan Derivatives in Drug Delivery. Advance Biochemistry Revised 5: 28-37.

Bot, Y.S. Mgbojikwe, L.O., Nwosu, C., Ambiku, A. and Damshak, D. (2007). Screening of the Fruit Pulp Extract of MomordicaBalsaminafor Anti HIV Property. African Journal. Biotechnology, 6(1): 47-52.

Dornard, A. and Rinaudo, M. (1983). Preparation and characterization of fully deacetylated chitosan. International Journal of Biological Macromolecules, 5: 49-52.

Dutta, P. K., Dutta, J. and Tripathi, V. S. (2004). Chitin and Chitosan: Chemistry, Properties and Applications. Journal of Scientific and Industrial Resources, 63: 20-31.

Elieh-Ali-Komi, D. and Hamblin, M. R. (2016). Chitin and Chitosan: Production and Application of Versatile Biomedical Nanomaterials, International Journal of Advanced Research, 4(3): 411-427.

Fernandez, S. and Kim, S. (2004). Physicochemical and Functional Properties of Crawfish Chitosan as Affected by Different Processing Protocols.

Hajjar, J., Habra, A. M. and Naing, A. (2013). Metformin: An Old Drug with New Potential. Expert Opinion on Investigational Drugs, 22 (12): 1511-1517.

Hassan, L. G. and Umar, K. J. (2006). Nutritional Value of Balsam Apple Leaves. Journal of Nutrition, 5:522-529.

Hossain, M. F. Chen, W., Yu, Zhang. (2015). Bulk Density of Mineral and Organic Soils in the anada's Artic and Sub-artic. Information Processing in Agriculture, 2(3-4): 183-190.

Jabbal-Gill, I., Watts, P. and Smith, A. (2012). Chitosan-Based Delivery Systems for Mucosal Vaccines. Expert Opinion onDrug Delivery, 9: 1051-1067.

Jiang, H.L., Park, I.K., Shin, N.R., Kang, S.G., Yoo, H.S., Kim, S.I., Suh, S.B., Akaike, T. and Cho, C.S. (2004). In Vitro Study of the Immune Stimulating Activity of an Atrophic Rhinitis Vaccine Associated to Chitosan Microspheres. European Journal of Pharmaceutical Biopharmacology, 58: 471-476.

Jiang, Y., Fu, C., Wu, S., Liu, G., Guo, J. and Su, G. (2017). Determination of the Deacetylation Degree of Chitooligosaccharides. Marine Drugs, 15: (11):332.

Kato, Y., Onishi, H. and Machida, Y. (2003). Application of Chitin and Chitosan Derivatives In the Pharmaceutical Field. Current Pharmaceutical Biotechnology, 4(5): 302-400.

Khan, T.A., Peh, K. K. and Chong, H.S. (2002). Reporting Degree of Deacetylation Values of Chitosan: The Influence of Analytical Methods. Journal of Pharmaceutical and Pharmacological Science, 5: 205-213.

Knorr, D. (1984). Use of Chitinous Polymers in Food -A Challenge for Food Research and Development. Food Technology Chicago, 38: 85-97.

Kumar, M. N.V.R. (2000). A Review of Chitin and Chitosan Applications", Reactive Functional Polymers, 46: 1-28.

Kumar, M., Tan, Zi. and John, D. A. (2017). Progress in Antimicrobial Activities of Chitin, Chitosan and its Oligosaccharides: A Systematic Study Needs For Food Applications. 
Kumar, N. P. B., Mahaboobi, S. and Satyam, S. (2017). Chitosan in Medicine: A Mini Review. Journal of Molecular Pharmaceutics and Organic Process Research, 5(1):134

Kumari, K. and Rani, U. (2011). Controlled Release of Metformin Hydrochloride Through Cross-Linked Blends of Chitosan-Starch. Advanced and Applied Science Residence, 2: 4854.

Kumirska, J., Czerwika, M., Kaczynski, Z., Bychowsks, A. and Brzozowski, K. (2010). Application of spectroscopic analysis of chitin and chitosan. Marine Drugs, 8:15671636

Li, Q., Dunn, E. T., Grandmaison, E.W. and Goosen, M. F. (1992). Applications and Properties of Chitosan. Journal of Bioactivity and Compativility of Polymer, 7: 370-397.

Mealey, B. L. and Oates, T. W. (2006). American Academy of Periodontology; Diabetes Mellitus and Periodontal Diseases. Journal of Period Ontology, 77: 1289-1303.

National Research Council Institute for Laboratory Animal research (NRC) (1996). Guide for the care and use of Laboratory Animals. National Academy of Sciences, Washington (DC).

No, H. K., Kim, S. D., Kim, D. S., Kim, S. J. and Meyers, S. P. (1999). Effect of Physical and Chemical Treatments on Chitosan Viscosity. Journal of Korean Society of Chitin and Chitosan, 4: 177-183.

Scheen, A. J. and Paquot, N. (2013). Metformin revisted: a critical review of the benefit-risk balance in at -risk patients with type 2 diabetes. Diabetes metabolism, 39 (3): 179-90

Shigemasa, Y., Matsuura, H., Sashiwa, H. and Saimoto H. (1996). Evaluation of different absorbance ratios from infrared spectroscopy for analyzing the degree of deacetylation in chitin. International Journal of Biological Macromolecules, 18:237-242.

Shoback, D. G. and Gardner, D. (2011). Greenspan's Basic and Clinical Endocrinology, 007162243-8.

Sikder, M. D. and Belal Hossain, M. S. M. (2019). Effect of Shrimp Chitosan Coating on Physicochemical Properties and Shelf Life. International Journal of Engineering Technology and Sciences (Ijets), https://doi.org/10.15282/Ijets.6.1.2019.1004

Sofowora, A. (1993). Medicinal Plants and Traditional in Africa. 2nd Ed. Sunshine House, Ibadan, Nigeria: Spectrum Books Ltd; Screening Plants for Bioactive Agents. Pp 134156

Trease, G. E. and Evans, W. C. (2002). Pharmacognosy. 15 th Ed London: Saunders Publishers, Pp 42-44. 221-229, 246-249,304-306, 331-332.

Yang, Q., Yuan, H., Chen, M., Qu, J., Wang, H., Yu, B., Chen, J., Sun, S., Teng, X.and Ren, W. (2018). Metfromin ameliorates the progression of atherosclerosis via supressing macrphage infilteration and inflammatory response in rabbits. Life science, 1(198): 5664 